\title{
STAR Forward Rapidity Upgrade
}

\author{
James D. Brandenburg* \\ Shandong University, Brookhaven National Laboratory \\ E-mail: jbrandenburgebnl.gov
}

The STAR Collaboration is designing, constructing, and installing a suite of new detectors in the forward rapidity region $(2.5<\eta<4)$ over the next two years, enabling a program of novel measurements in $\mathrm{p}+\mathrm{p}, \mathrm{p}+\mathrm{A}$, and $\mathrm{A}+\mathrm{A}$ collisions. This extension of STAR's kinematic reach will allow detailed studies of cold QCD physics at both very high and very low partonic momentum fraction, i.e., when the colliding quarks and gluons carry very large or very small amounts of the nucleon energy. To fully explore some of the outstanding QCD physics opportunities, the forward upgrade has detection capability for neutral pions, hadrons, photons, electrons, jets, and adds charged-particle tracking, electromagnetic, and hadronic calorimetry to STAR's capabilities at high pseudorapidity. The upgrade will greatly expand the kinematic reach for ongoing measurements of the spin and flavor structure of the nucleon and will enable studies of the longitudinal structure of the nuclear initial state that leads to breaking of boost invariance in heavy-ion collisions. Transport properties of the hot and dense matter formed in heavy-ion collisions will also become accessible with the new measurement capabilities at forward rapidity.

HardProbes 2020

1-6 June 2020

Austin, Texas

${ }^{*}$ Speaker. 


\section{Physics Opportunities at Forward Rapidity}

As outlined in the RHIC Cold QCD Plan [1], precise imaging of gluons and sea quarks inside protons and nuclei is needed to address some of the deepest questions about the emergence of nuclear properties from QCD. Some of the important outstanding questions include: How are the gluons and sea quarks distributed in space and momentum inside the nucleon; How do the spin of gluons and sea quarks (and their orbital angular momentum) contribute to the total nucleon spin; Does the density of gluons saturate inside nuclei at high energy, and if so, what are the universal properties of saturated gluonic matter? Answering these questions and others about the fundamental aspects of QCD have motivated the proposed electron-ion collider (EIC) [2, 3], the next-generation experiment for studying the constituents of nuclear matter in detail. The STAR forward rapidity physics program offers opportunities to address these and other outstanding questions in cold QCD in the years leading up to the EIC and paves the way for the physics program of the future EIC.
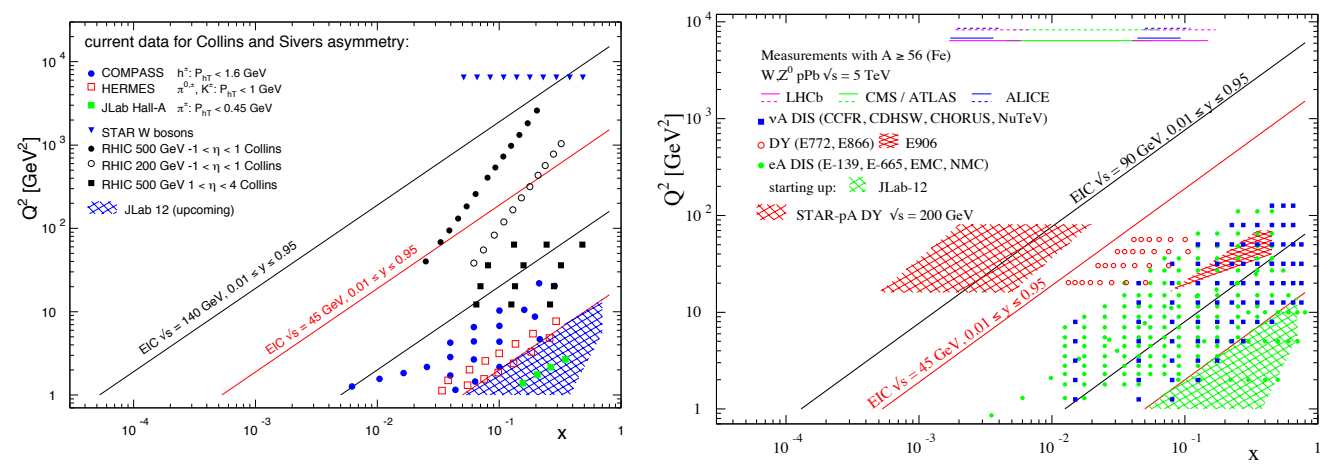

Figure 1: Left: The $x-Q^{2}$ plane with data sensitive to the Collins and Sivers asymmetry from all current semi-inclusive deep inelastic scattering measurements and future EIC and JLAB-12 GeV projections. The unique region of $x$ and $Q^{2}$ that will be covered by STAR measurements at mid and forward rapidity are shown. Right: the $x-Q^{2}$ plane depicting the measurements sensitive to nuclear parton distribution functions, including predictions for the future EIC measurements and STAR measurements at forward rapidity in $\mathrm{p}+\mathrm{A}$ collisions at $\sqrt{s}=200 \mathrm{GeV}$.

Previous STAR efforts employing the forward pion detector and the forward meson spectrometer have demonstrated that important questions in cold QCD physics can be addressed by STAR with detailed measurements in the forward rapidity region. New forward instrumentation will allow STAR to perform novel measurements of the large transverse single-spin asymmetry, $A_{N}$, observed in charged hadrons [4]. Measurement of $A_{N}$ at the highest RHIC center-of-mass energies will allow tests of the leading theoretical descriptions $[5,6]$ and provide novel data for determining the underlying mechanism of this observed asymmetry [7, 8]. The unique flexibility of the RHIC complex allows the collision of polarized proton beams. Measurement of transversely polarized proton collisions at forward rapidity may help to develop a more complete picture of the nucleon spin structure $[9,10]$. STAR Measurements in the forward region of the Collins and di-hadron asymmetry, observables sensitive to transversity, would provide data in unexplored $x$-ranges and allow investigation of the flavor dependence of transversity. Figure 1 (left) shows the $x-Q^{2}$ coverage 
that will be possible with the RHIC measurements compared to those from the future EIC, JLab-12, and the current semi-inclusive deep inelastic scattering world data.

Precision measurements of nuclear parton distribution functions (nPDFs) at small $x$ are needed to constrain the initial state of A+A collisions [11]. As shown in Fig. 1 (right), forward instrumentation will allow STAR to probe the moderate $Q^{2}$ and medium-to-low $x$ range where no data currently exist - in a region where the nuclear modification of sea quarks and gluons is expected to be sizable. The use of forward calorimetry to enable electron identification and hadron rejection will allow measurement of the nuclear modification of sea quarks through the $R_{p A}$ of Drell-Yan, which is ideal for the measurement of sea quark suppression since it is free from final state effects. Similarly, the combination of charged particle tracking and electromagnetic calorimeters will allow measurement of direct photon suppression at forward rapidity, which is an ideal probe sensitive to the expected gluon suppression at low $x$.

While the primary physics motivation for the STAR forward rapidity physics program is the exploration of cold QCD physics in the very high and low regions of Bjorken $x$, the detector suite needed to address these topics will also provide new detector capabilities to STAR for the exploration of timely topics in hot QCD as well. The greatly expanded kinematic range of the STAR detector will allow measurements that are sensitive to the longitudinal structure of the initial state and the temperature dependent transport properties of matter in relativistic heavy-ion collisions. Measurements of long-range correlations are expected to be sensitive to the early-time dynamics of heavy-ion collisions. Forward instrumentation complements the existing STAR detectors, providing an expanded two-particle phase space for measuring long range correlations.

In order to fully explore these topics in both cold and hot QCD, the STAR forward rapidity physics program has been designed to provide superior detection capability for neutral pions, photons, electrons, jets, and hadrons through the addition of charged-particle tracking and electromagnetic and hadronic calorimetry to STAR in the forward rapidity region. Figure 2 shows a render of the STAR detector, including the forward tracking system and the forward calorimeter system.

\section{The STAR Forward Rapidity Upgrade}

Achieving the physics goals of the STAR forward rapidity physics program requires the design, construction, and installation of several new detector subsystems into STAR in the forward region. The forward tracking system (FTS) will provide charged particle tracking while the forward calorimeter system (FCS) will provide electromagnetic and hadronic calorimetry. Both the FTS and the FCS will cover the region $2.5<\eta<4.0$. The FTS must be capable of discriminating the charge sign of hadrons for transverse asymmetry studies and be capable of separating electrons from positrons for Drell-Yan measurements. The physics goals for measurements in A+A collisions, such as the two-particle correlation measurements, further require a momentum resolution better than $30 \%$ for tracks with $0.2<p_{T}<2 \mathrm{GeV} / c$ and an efficiency of better than $80 \%$ for events with approximately 100 tracks per event.

\subsection{The Forward Tracking System}

The FTS consists of a combination of two detector technologies: three stations of silicon 


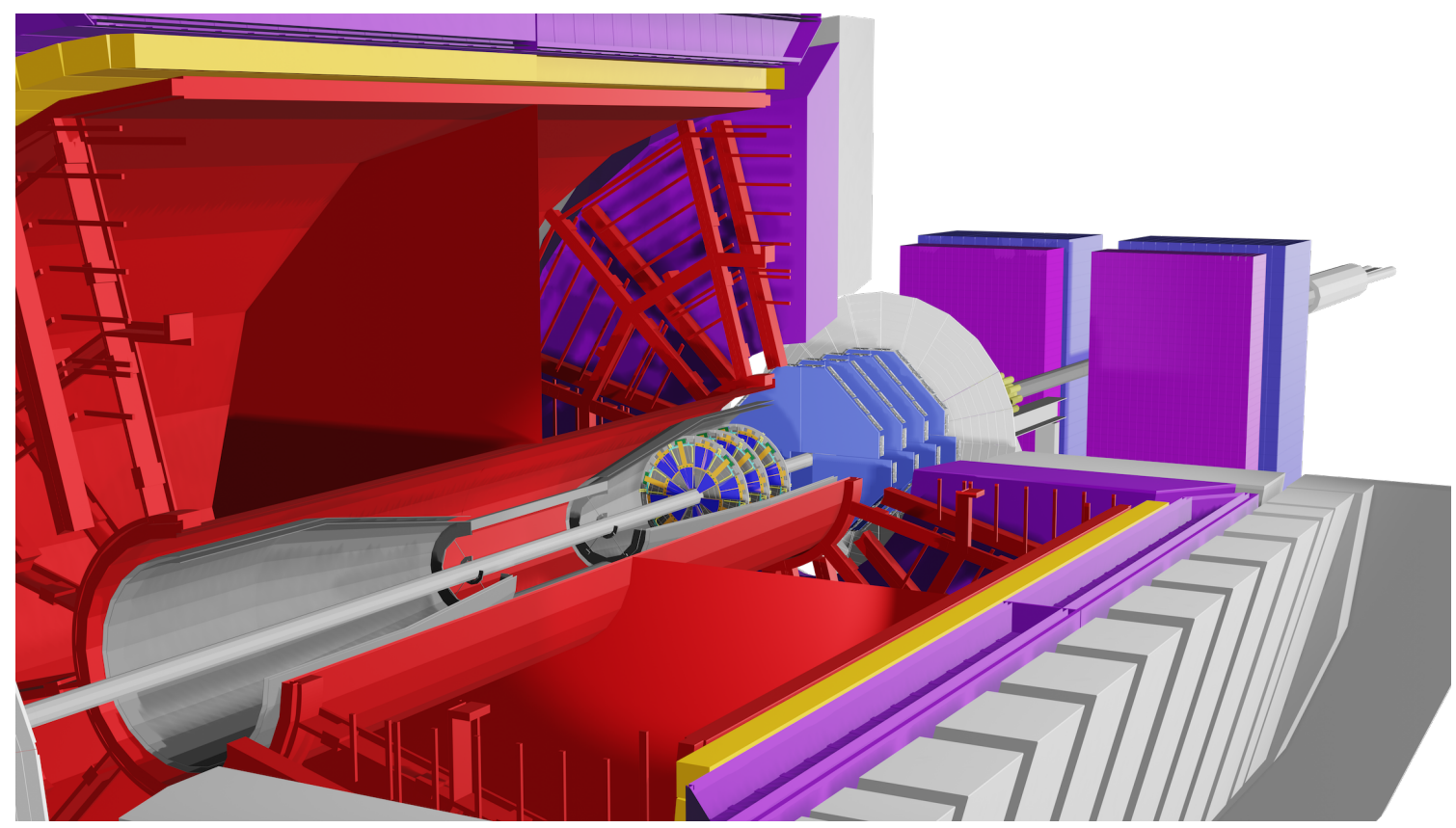

Figure 2: A render of the STAR detector, including the forward tracking system and the forward calorimeter. The silicon mini-strip disk detectors are shown closest to the interaction point, followed by the pentagonal sTGC detectors. The event plane detector (grey disk) is used as a preshower detector for the forward calorimeter system (purple and blue).

mini-strip sensors and four stations of small-strip thin gap chambers (sTGCs) similar to those used in the ATLAS new small wheel upgrade[12,13]. As depicted in Fig. 2, the silicon detectors are placed nearest to the interaction point at $z$-locations of approximately $140 \mathrm{~cm}$ to $200 \mathrm{~cm}$ from the interaction point. The sTGCs are placed further away at $z$-locations between $300 \mathrm{~cm}$ to $360 \mathrm{~cm}$ from the interaction point inside the magnet pole tip opening. While the silicon sensors lie within the region of the homogeneous 0.5 T STAR magnetic field, the STGC are in a region where the field changes gradually, making charged particle tracking more involved.

The silicon mini-strip detector design and electronic systems take advantage of the experience gained with the intermediate silicon tracker (IST) [14] used in STAR previously. The forward silicon mini-strip detectors use silicon sensors from Hamamatsu, APV25-S1 frontend readout chips, flexible hybrids, and provide a total material budget of only $\approx 1.5 \%$ per disk. Each silicon disk is made up of 12 modules with each module segmented coarsely in $R$ but finely in $\phi$ (with 128 strips per module). The DAQ and cooling systems also make use of previous systems developed for the IST. Each of the sTGC detectors is a combination of four quadrants made of two double sided chambers each. A symmetric pentagonal design is used to allow maximum reuse of tooling and to simplify module design. Each detector includes $\mathrm{X}, \mathrm{Y}$ and one layer of diagonal strips for hit location measurement. A dedicated gas system has been designed which uses controlled evaporative mixing to provide the detector with a gaseous mixture of $55 \%$ n-pentane $+45 \% \mathrm{CO}_{2}$. VMM-base readout electronics are used for the sTGC following the ATLAS design. 


\subsection{The Forward Calorimeter System}

The FCS is a crucial subsystem needed for several of the physics measurements outlined above. In order to achieve the proposed physics goals, the electromagnetic calorimeter must provide energy resolution of $\sigma / E \approx 10 \% / \sqrt{E}$ and the hadronic calorimeter must provide energy resolution of $\sigma / E \approx 50 \% / \sqrt{E}+10 \%$. The FCS is located about $7 \mathrm{~m}$ from the interaction point. The electromagnetic calorimeter (ECAL) makes use of refurbished PHENIX lead-scintillator $(\mathrm{PbSc})$ with new SiPM-based readout electronics on the front face. Each PbSc tower is $5.52 \times 5.52 \times 33$ $\mathrm{cm}^{3}\left(\sim 18 X_{0}\right)$ with sampling cells composed of $1.5 \mathrm{~mm} \mathrm{~Pb}, 4 \mathrm{~mm}$ scintillator \& wavelength shifting fibers. The ECAL has 1,496 readout channels. The hadronic calorimeter (HCAL) system is an iron-scintillator $(\mathrm{FeSc}$ ) sandwich sampling calorimeter with each sandwich composed of $20 \mathrm{~mm} \mathrm{Fe}$ and $3 \mathrm{~mm}$ scintillator. The lateral tower size for the HCAL towers is $10 \mathrm{~cm} \times 10 \mathrm{~cm}$. Together, the ECAL+HCAL provide approximately 5.2 $\lambda$. The HCAL uses the same SiPM-based readout as the ECAL with a total of 520 readout channels. As part of the forward calorimeter system, the existing event plane detector is used as a preshower detector. The FCS system provides triggering capabilities for the entire forward detector system. ECAL and HCAL prototypes were tested with beam at Fermi National Accelerator Laboratory and their performance was found to be approximately within the requirements.

\section{Summary}

STAR has undertaken an upgrade to add charged particle tracking and calorimetry in the forward rapidity region, $2.5<\eta<4$. , in order to address important topics in both cold and hot QCD. The installation of the forward calorimeter system is scheduled for completion by early 2021 and the installation of the forward tracking system is scheduled for completion in the fall/winter of 2021. Upon completion of the forward detectors, STAR will collect data from transversely polarized $p+p$ collisions at $\sqrt{s}=510 \mathrm{GeV}$. Additional measurements of $\mathrm{p}+\mathrm{p}, \mathrm{p}+\mathrm{A}$, and $\mathrm{A}+\mathrm{A}$ collisions at $\sqrt{s_{N N}}=200 \mathrm{GeV}$ are expected to take place in 2023+. The combination of STAR's new forward rapidity detectors, along with the recently completed upgrades at mid-rapidity for the beam energy scan II, provide STAR with a unique opportunity to engage in novel measurements capable of answering important open questions in both hot and cold QCD.

\section{References}

[1] E.-C. Aschenauer, C. Aidala, A. Bazilevsky, M. Diehl, R. Fatemi, C. Gagliardi et al., The RHIC Cold QCD Plan for 2017 to 2023: A Portal to the EIC, arXiv:1602.03922 [nucl-ex] (2016) .

[2] E.C. Aschenauer, S. Fazio, J.H. Lee, H. Mäntysaari, B.S. Page, B. Schenke et al., The Electron-Ion Collider: Assessing the Energy Dependence of Key Measurements, arXiv:1708.01527 [hep-ex, physics:hep-ph, physics:nucl-ex, physics:nucl-th] (2017).

[3] A. Accardi, J.L. Albacete, M. Anselmino, N. Armesto, E.C. Aschenauer, A. Bacchetta et al., Electron Ion Collider: The Next QCD Frontier - Understanding the glue that binds us all, arXiv:1212.1701 [hep-ex, physics:hep-ph, physics:nucl-ex, physics:nucl-th] (2014).

[4] BRAHMS Collaboration, I. Arsene, I.G. Bearden, D. Beavis, S. Bekele, C. Besliu et al., Single-transverse-spin asymmetries of identified charged hadrons in polarized pp collisions at $\sqrt{s}=62.4$ gev, Phys. Rev. Lett. 101 (2008) 042001. 
[5] J. Collins and T. Rogers, Understanding the large-distance behavior of transverse-momentum-dependent parton densities and the Collins-Soper evolution kernel, Phys. Rev. D 91 (2015) 074020.

[6] K. Kanazawa, Y. Koike, A. Metz and D. Pitonyak, Towards an explanation of transverse single-spin asymmetries in proton-proton collisions: The role of fragmentation in collinear factorization, Phys. Rev. D 89 (2014) 111501.

[7] A.V. Efremov and O.V. Teryaev, QCD asymmetry and polarized hadron structure function measurement, Physics Letters B 150 (1985) 383.

[8] J. Qiu and G. Sterman, Single transverse spin asymmetries, Phys. Rev. Lett. 67 (1991) 2264.

[9] A.I. Signal, Calculations of higher twist distribution functions in the MIT bag model, Nuclear Physics B 497 (1997) 415.

[10] M. Radici and A. Bacchetta, First Extraction of Transversity from a Global Analysis of Electron-Proton and Proton-Proton Data, Phys. Rev. Lett. 120 (2018) 192001.

[11] N. Armesto, H. Paukkunen, J.M. Penín, C.A. Salgado and P. Zurita, An analysis of the impact of LHC Run I proton-lead data on nuclear parton densities, Eur. Phys. J. C 76 (2016) 218.

[12] G. Sekhniaidze, Construction and performance of the sTGC and MicroMegas chambers for ATLAS NSW upgrade, J. Inst. 12 (2017) C03020.

[13] X. Zhao, W. Li, D. Zhang, C. Li, H. Li, S. Liu et al., Cosmic test of sTGC detector prototype made in China for ATLAS experiment upgrade, Nuclear Instruments and Methods in Physics Research Section A: Accelerators, Spectrometers, Detectors and Associated Equipment 927 (2019) 257.

[14] B. Buck, E. Anderssen, J. Bessuille, M. Cepeda, T. Johnson, J. Kelsey et al., Design and Fabrication of a Highly Integrated Silicon Detector for the STAR Experiment at Brookhaven National Laboratory, arXiv:1404.0993 [physics] (2014) . 